Document downloaded from:

http://hdl.handle.net/10251/157307

This paper must be cited as:

Kerrache, CA.; Lagraa, N.; Hussain, R.; Ahmed, SH.; Benslimane, A.; Tavares De Araujo Cesariny Calafate, CM.; Cano, J.... (2019). TACASHI: Trust-Aware Communication Architecture for Social Internet of Vehicles. IEEE Internet of Things. 6(4):5870-5877. https://doi.org/10.1109/JIOT.2018.2880332

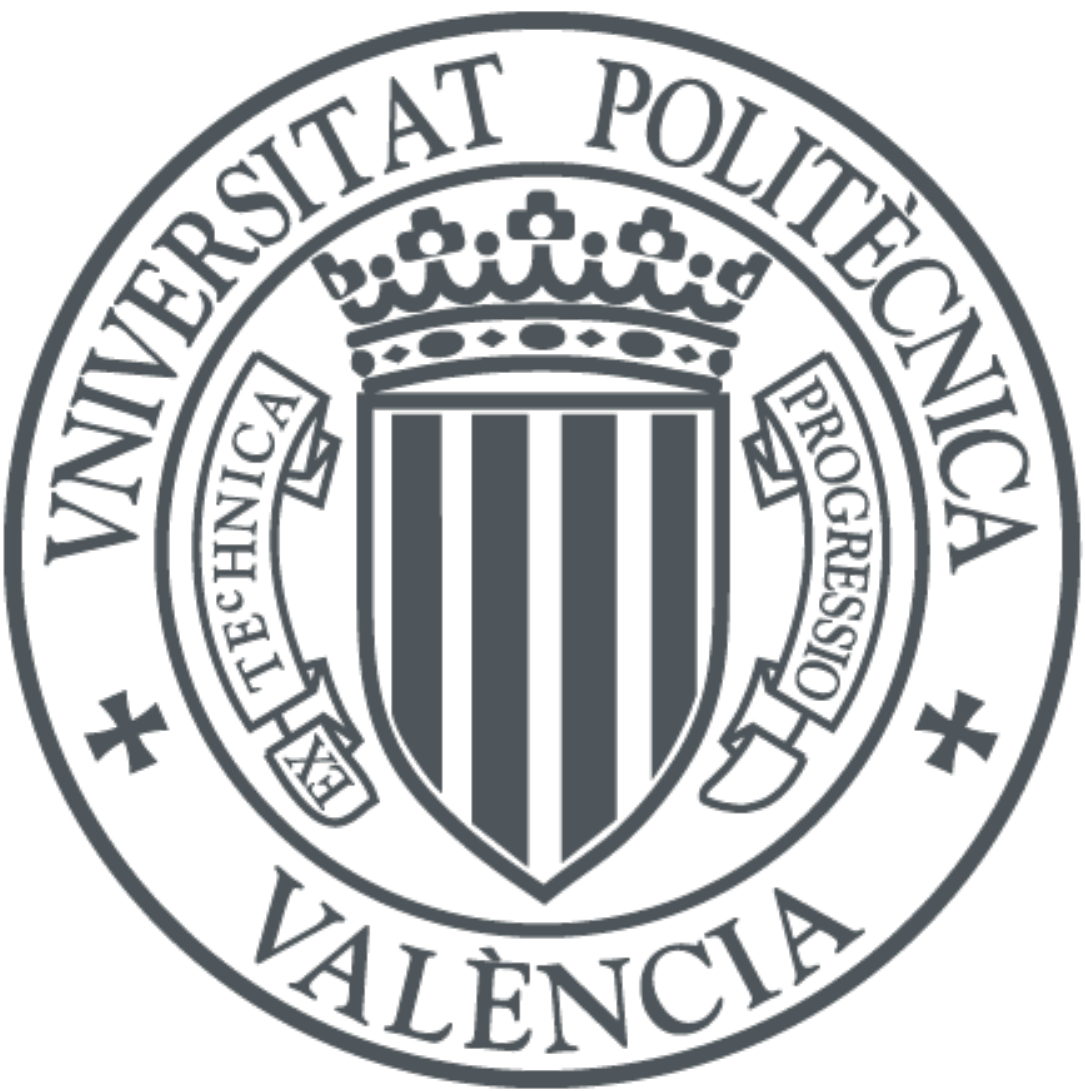

The final publication is available at

https://doi.org/10.1109/JIOT.2018.2880332

Copyright Institute of Electrical and Electronics Engineers

Additional Information 


\title{
TACASHI: Trust-Aware Communication Architecture for Social Internet of Vehicles
}

\author{
Chaker Abdelaziz Kerrache ${ }^{(}$, Nasreddine Lagraa, Rasheed Hussain ${ }^{(}$, Syed Hassan Ahmed ${ }^{(}$, \\ Abderrahim Benslimane $^{(\mathbb{0}}$, Carlos T. Calafate $^{\mathbb{(}}$, Juan-Carlos Cano, and Anna Maria Vegni ${ }^{(\mathbb{C}}$
}

\begin{abstract}
The Internet of Vehicles (IoV) has emerged as a 2 new spin-off research theme from traditional vehicular ad hoc 3 networks. It employs vehicular nodes connected to other smart 4 objects equipped with a powerful multisensor platform, commu5 nication technologies, and IP-based connectivity to the Internet, 6 thereby creating a possible social network called Social IoV (SIoV). Ensuring the required trustiness among communicating s entities is an important task in such heterogeneous networks, 9 especially for safety-related applications. Thus, in addition to to securing intervehicle communication, the driver/passengers hon11 esty factor must also be considered, since they could tamper 12 the system in order to provoke unwanted situations. To bridge 3 the gaps between these two paradigms, we envision to connect ${ }_{4}$ SIoV and online social networks (OSNs) for the purpose of estimating the drivers and passengers honesty based on their ${ }_{16}$ OSN profiles. Furthermore, we compare the current location of 17 the vehicles with their estimated path based on their historical 18 mobility profile. We combine SIoV, path-based and OSN-based 19 trusts to compute the overall trust for different vehicles and their 20 current users. As a result, we propose a trust-aware communi21 cation architecture for social IoV (TACASHI). TACASHI offers 22 a trust-aware social in-vehicle and intervehicle communication 23 architecture for SIoV considering also the drivers honesty factor 24 based on OSN. Extensive simulation results evidence the effi25 ciency of our proposal, ensuring high detection ratios $>87 \%$ and 26 high accuracy with reduced error ratios, clearly outperforming 27 previous proposals, known as RTM and AD-IoV.
\end{abstract}

Index Terms-Human factor, Social Internet of Vehicles (SIoV), 29 trust, vehicular ad hoc network (VANET).

30

31

$" \mathrm{M}$ ANY applications have been realized through vehicular networks as a result of communication among

C. A. Kerrache is with the Department of Mathematics and Computer Science, University of Ghardaia, Ghardaïa 47000, Algeria (e-mail: ch.kerrache@lagh-univ.dz).

N. Lagraa is with the University of Laghouat, Laghouat 03000, Algeria (e-mail: n.lagraa@lagh-univ.dz).

R. Hussain is with the Institute of Information Systems, Innopolis University, 420500 Innopolis, Russia (e-mail: r.hussain @innopolis.ru).

S. H. Ahmed is with the Department of Computer Science, Georgia Southern University, Statesboro, GA 30460 USA (e-mail: sh.ahmed@ieee.org).

A. Benslimane is with the University of Avignon, 84911 Avignon, France (e-mail: abderrahim.benslimane@univ-avignon.fr).

C. T. Calafate and J.-C. Cano are with the Department of Computer Engineering, Universitat Politècnica de València, Valencia, Spain (e-mail: calafate@disca.upv.es; jucano@disca.upv.es).

A. M. Vegni is with the Department of Engineering, Roma Tre University, 00146 Rome, Italy (e-mail: annamaria.vegni@uniroma3.it). vehicles and/or the infrastructure [1]. These applications are 33 abstractly classified into safety and nonsafety related applications. The former class of applications exhibit stringent requirements, such as delay-critical, security-critical, trustcritical, and decision-critical features, whereas the latter class of applications have relatively less stringent requirements. Nevertheless, many of these applications represent a decisionaided system where the final decision (usually taken by the human drivers) have a direct effect on the outcome of the decision. Therefore, the trustworthiness of the information and the source of information is of prime importance.

In Internet of Vehicles (IoV) paradigm, each vehicle is considered as a smart object equipped with a powerful multisensor platform, communications technologies, computation units, IP-based connectivity to the Internet, and to other vehicles either directly or indirectly. In addition, a vehicle in IoV is envisioned with a multicommunication model, enabling the interactions among intravehicle components, intervehicles, vehicle-to-infrastructure, and vehicles-to-people. IoV also 51 enables the acquisition and processing of large amount of data 52 from versatile geographical areas via intelligent vehicles com- 53 puting platforms, to offer various categories of services for 54 road safety and other services to drivers and passengers [2].

To this end, the communication of vehicles with differ- 56 ent entities in IoV exhibit social features at par with the traditional social networks where the nodes share information. More precisely, Social IoV (SIoV) are a breed of ${ }_{59}$ socially aware ephemeral networks [3], where vehicular nodes 60 share/exchange information with different entities and thus- 61 forth comparable with the traditional social networks. On the 62 other hand, with the emergence of $5 \mathrm{G}$ technology, almost all 63 Internet services can be accessed anytime and anywhere [4]. 64 In addition, vehicles' mobility patterns can be easily estimated 65 through its history profiles and the drivers' social interactions 66 and hobbies. Hence, the SIoV system can trigger a possible 67 event, which would advocate for verification of the situation, 68 resulting in stolen vehicle alert an alert or even, text the vehicle's owner. It is indeed possible that there could be false alarms; however, more insights are needed to this issue.

To fill the gaps, in this paper, we propose a novel SIoV com- 72 munication architecture that takes advantage of online social 73 networks (OSNs) to enhance the SIoV trust establishment by 74 considering the human and location-related honesty (LRH). 75 We leverage the group-trust metric adopted by Advogato, ${ }^{1}{ }_{76}$

${ }^{1}$ [Online]. Available: http://www.advogato.org/ 34 35 36 8 39 40 41 42

.

.

61

62

4

65

66

.


77 attempting to determine the maximum set of trusted peers, 78 while minimizing the influence of unreliable dishonest peers 79 during communication [5]. Afterward, an honesty-related clas80 sification (i.e., good, bad, or compromised) is associated to 81 every node (driver/passenger) and vehicle location depending 82 on the Advogato classification of this node (i.e., either trusted 83 or distrusted) and the location tracking system, respectively. ${ }_{84}$ In addition, in-vehicle interdevice communications are secured 85 using a lightweight technique based on Chaotic Maps.

Furthermore, the intervehicle trust is also estimated, 87 combined with the discrete recommendations from RSUs 88 and trusted authorities (TAs). Finally, the Advogato results 89 are used to probabilistically identify honest and dishonest 90 drivers/passengers. Using this strategy, the aim is not just to 91 reduce both the detection error ratios and also the ratio of 92 doubtful nodes that the intervehicle trust could not classify 93 them to either trusted or distrusted peers but also to prevent 94 unwanted situations, such as stolen vehicles thanks to the LRH 95 estimation.

To summarize, the contributions of this paper are as follows.

1) We propose a trust-aware communication architecture for social IoV (TACASHI), which offers a trust-aware social in-vehicle and intervehicle communication architecture for SIoVs.

2) Secure in-vehicle communications are guaranteed through Chaotic Maps.

3) Drivers' honesty consideration using their OSN profiles reached through a trusted middleware.

4) Vehicles movement-related honesty estimation through the use of their historical mobility patterns and a path prediction algorithm.

108 The remainder of this paper is organized as follows. In 109 Section II, we present some background in vehicular ad hoc 110 network (VANET), IoV, OSNs, and trust establishment in both 111 kinds of networks. Afterward, in Section III, we present an 112 overview of our proposal, followed by its details in Section IV. ${ }_{113}$ TACASHI's dishonesty detection process is then discussed in 114 Section V. Section VI presents our simulation environment, 115 followed by the discussion of the results obtained. Finally, the 116 conclusions are drawn at the end of this paper.

\section{STATE-OF-THE-ART}

118 Trust establishment in vehicular networks is essential for 119 the realization of efficient secure applications. Various solu120 tions have adopted trust modeling to enhance the intervehicle 121 communications for VANETs, IoV, and SIoV. In this section, 122 we provide an overview of the main features of socially aware 123 networking, as well as the existing trust-based solutions in 124 these domains.

\section{${ }_{125}$ A. Social Trust and Socially Aware Networking}

126 The proliferation of hand-held devices demands mobile car127 riers to provide instant connectivity. Moreover, the movements 128 of the users are generally related to their social behaviors and 129 relationships, and the mobility patterns of mobile devices car130 ried by these users are strongly coupled with their movements. 131 Thus, mobile networks are nowadays more human-centric. As a result, a new field called socially aware networking has sur- 132 faced that takes the human behavior into account [6]. This new 133 paradigm of social-awareness is applicable to many types of 134 internode interaction-based networks, such as ad hoc networks 135 and its different breeds.

\section{B. Trust in OSNS}

As aforementioned, trust establishment is primarily impor- 138 tant for enhancing the security of different networks and 139 many solutions used trust establishment mechanisms for 140 OSNs [7], [8]. The general trust establishment solutions for 141 OSNs are based on either Advogato trust metric [5] or 142 PageRank-based solutions [9].

Generally, trust for OSNs can be classified using three com- 144 plementary phases: 1) trust information collection; 2) trust 145 evaluation; and 3) trust information dissemination. To identify 146 how honest and trustful is a profile owner, social trust is based ${ }_{147}$ on a scalar estimation using the personal profile information, 148 which includes user identity and interactions with other users. 149 Once this social trust is estimated, it will be provided to the 150 end users in different forms and for different purposes.

\section{Trust in VANETs and IoV}

In the VANET context, trust management schemes are gen- 153 erally classified as entity-based, content-based, and hybrid 154 models following the targeted adversary, which can be dis- 155 honest entities, malicious messages, or both [10]. Several 156 works in the literature addressed entity-based trust models. ${ }_{157}$ Yang's [11] solution is based on revocation of the nodes that 158 sent falsified or fake information using different techniques. 159 Haddadou et al. [12] chose to associate a credit value to 160 each neighbor vehicle that will increase or decrease depend- 161 ing on the messages credibility of the concerned neighbor's. 162 Hence, this credit will be quickly decreased when replaying 163 or injecting new (potentially false or malicious) messages. ${ }_{164}$

For content-based trust management, Gurung et al. [13] 165 adopted three metrics to classify the received messages into 166 either legal or malicious messages; these metrics are con- 167 tent similarity, content conflict, and routing path similarity. 168 However, in addition to its high time complexity, this solution 169 does not take into account the high level of mobility exhibited 170 by VANET nodes and the node sparsity. On the other hand, 171 our previous hybrid models [14], [15] focus mainly on facing 172 denial-of-service and coalition attacks in VANETs using the 173 standardized messaging service. However, the additional traf- 174 fic generated by the recommendation requests/responses might 175 affect some safety-related applications. Additionally, few solu- 176 tions addressing trust issues in the IoV have also been recently 177 published [16].

Hossain et al. [17] proposed a trust model for collect- 179 ing evidence from IoV infrastructures, store them in vehicles 180 tamper-proof devices, and then start intervehicle trust-based 181 communication. The main limitation of such approach is that 182 the behavior of vehicles may change. Thus, trust information 183 values should remain static over time. In addition, authors 184 did not evaluate the performance in a realistic environment 185 implementing the different low-layer features of VANETs. $\quad 186$ 
187 Unlike existing trust models, Gai et al. [18] proposed a 188 trust management system for SIoV called RTM where each 189 node stores its own reputation information rated by others 190 during past transactions. They introduced a CA server to 191 ensure the integrity and the undeniability of the trust informa192 tion. However, besides the additional cost of the introduced 193 server, this scheme may not be effective in rural scenarios or 194 low-density scenarios. Furthermore, as like in other existing 195 solutions, the human honesty factor is not considered.

\section{D. Trust Computation in Vehicular Networks and OSNs}

197 Due to the distributed and ephemeral nature of vehicu198 lar networks, every vehicle locally evaluates its neighbors' 199 trust. This trust computation can be carried out either in a 200 scalar way, using the piggybacked opinions within exchanged 201 messages, or through clustered and group-based collaboration 202 among vehicles located in a same area [19]. Whereas, trust 203 in OSNs requires having a sink or a third trusted party who 204 is responsible for evaluating the trust for different peers. This 205 sink can either handle the whole task of trust computation, or 206 it can distribute such task among secondary sinks, which are 207 typically community leaders [20].

208 In the light of the existing works, there is a still a huge 209 gap between the requirements of the trust-based communi210 cation in SIoV and the existing solutions. To fill the gaps, 211 we propose a novel trust-based SIoV communication archi212 tecture (namely, TACASHI), which besides the intervehicle 213 trust establishments evaluates also their drivers and move214 ment honesty. Furthermore, TACASHI also offers a secure and 215 lightweight in-vehicle communication strategy.

\section{III. TACASHI OVERVIEW}

217 Establishing SIoV trust with the incorporation of the human 218 honesty factor should be achieved by relying on third TAs 219 as intermediaries for this information, since these authorities 220 are the only ones having the possibility to trace/track vehi221 cles identities together with their drivers/owners. Accounting 222 for the vehicles' identity is not a problem, as every vehi223 cle should have a valid certificate and a set of pseudonyms ${ }_{224}$ provided by the TA. However, matching the driver identity 225 and social account with the vehicle identity involves the use 226 of other intermediate tools, such as digital fingerprint, eyes 227 and voice recognition systems, or a subscriber identification 228 module, thus imposing more requirements onto the system.

229 Due to the high cost of smart vehicles, and to the probable 230 lack of RSUs in rural environments, Android-based platforms, 231 including smartphones and tablets have recently emerged as 232 an alternative solution to provide vehicular communications. ${ }^{2}$ 233 This way, any trusted third authority can be reached using 234 different cellular network technologies. This new research area 235 is know as heterogeneous vehicular networking [21].

Fig. 1 represents an overview of our proposed SIoV archi237 tecture in which, besides passengers, vehicles, roadside units, 238 and TAs, we also involve OSNs. The latter are accessed

\footnotetext{
${ }^{2}$ The SmartCarPhone project. [Online]. Available: http://www.grc.upv.es/ SmartCarPhone/
}

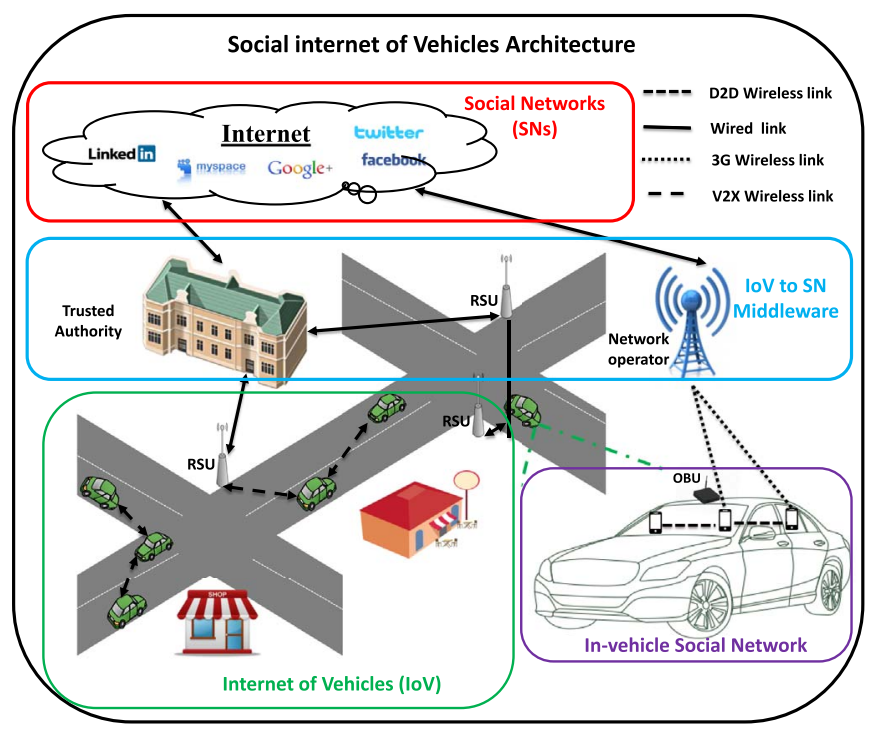

Fig. 1. Proposed SIoV architecture.

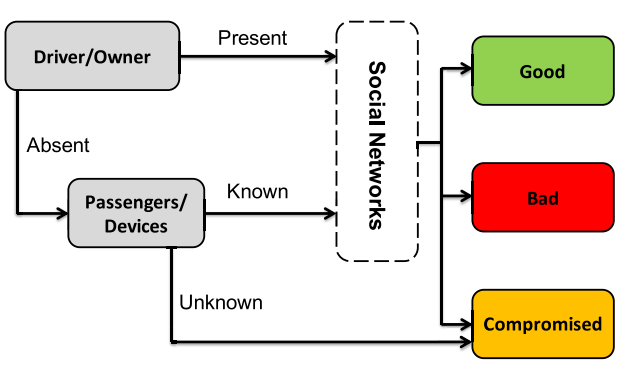

Fig. 2. Driver and passengers honesty factor.

through a trusted middleware provided by the network opera- 239 tor, RSUs, or TA like the City Hall.

TACASHI architecture involves five main actors: 1) the per- 241 son registered as the vehicle owner; 2) the passengers within 242 the vehicle represented by their connected devices; 3) the 243 vehicles themselves; 4) road side units and TAs; and 5) the 244 OSN accounts connected to the driver and passengers' devices. 245 In addition, a path prediction algorithm [22] is also used to 246 estimate and judge the current vehicle locations.

\section{TACASHI'S TRUST ESTABLISHMENT}

248

As mentioned in the previous sections, our proposal involves 249 drivers' honesty (see Fig. 2), vehicles' honesty (see Fig. 3), 250 and vehicles' LRH (see Fig. 4). Before detailing how these 251 factors are computed in the following sections, the next 252 section presents the proposed in-vehicle interdevice secure 253 communication process.

$$
254
$$

\section{A. In-Vehicle Interdevices Authentication Process}

255

In order to enable OSN-based trust, while preserving 256 drivers/owners privacy, the department of motor vehicles 257 (DMV) initializes the OBU by performing a number of oper- 258 ations. First, the driver enters its anonymized OSN account 259 and the DMV registers it against the user. DMV also issues a 260 number of pseudonyms to user $a$, i.e., $\left\{\mathrm{ID}_{1}^{a}, \mathrm{ID}_{2}^{a}, \ldots, \mathrm{ID}_{n}^{a}\right\}$. 


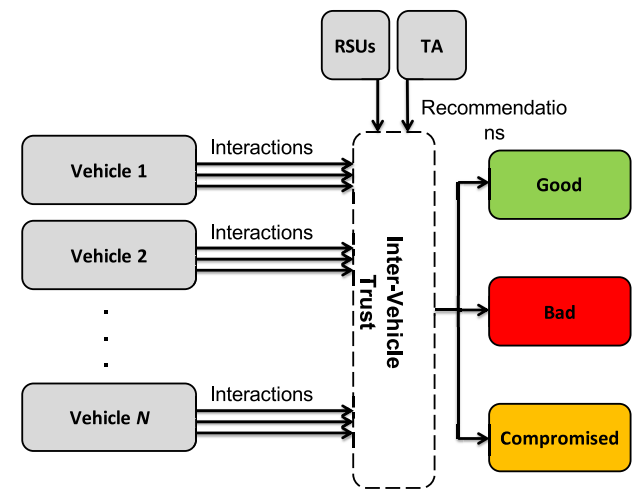

Fig. 3. Vehicles honesty factor.

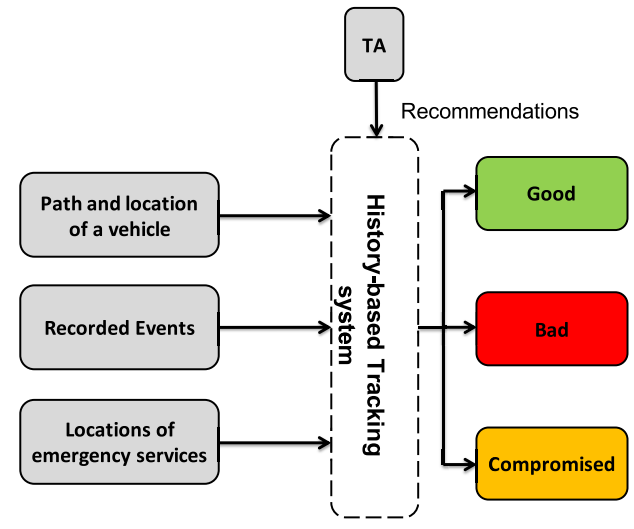

Fig. 4. LRH factor.

262 In-vehicle device/passengers in TACASHI are required to 263 pass the authentication process before gaining access to the 264 different network operations. If these devices fail to be authen265 ticated, they are directly classified as compromised devices, as 266 shown in Fig. 2.

267 We assume that all the devices in a network have an identity ${ }_{268}\left(\mathrm{ID}_{i}\right)$, and get the secure token from the TA; this token is 269 assumed to be received through a secure channel. All the nodes 270 compute the public key $(x, T k(x))$ and private key $k$ using ${ }_{271}$ Chaotic Maps based on Chebyshev polynomials, which are 272 known to be less energy consuming than RSA and ECC [23].

${ }_{273}$ Consider the communication between devices $\mathrm{A}$ and $\mathrm{B}$ 274 with their identities, i.e., $\operatorname{ID}_{a}$ and $\operatorname{ID}_{b}$, and their public and 275 private key pairs are $\left\{\left(x, T k_{a}(x)\right), k_{a}\right\}$ and $\left\{\left(x, T k_{b}(x)\right), k_{b}\right\}$, 276 respectively.

277 If node A wants to securely communicate with node B, it 278 initiates the authentication request as follows.
1) Node A selects a prime number $p$ and computes the value of $T_{p}(x)$.

2) Node A sends the message $m a=\left\{H_{a}, C_{a}\right\}$ to node $\mathrm{B}$.

3) After getting the message $m a=\left\{H_{a}, C_{a}\right\}$ from node A, B decrypts $C_{a}$ with the key $k=T_{t}(x)$ received from TTP, and compares the value of PW from the decrypted message with its obtained PW value from TTP. If there is a match, then node $\mathrm{B}$ concludes that $\mathrm{A}$ is an authenticated node.

4) Afterward, it checks the message integrity by computing the hash value, and compares it with $H_{a}$. If there is a match, then B concludes that the message was not 290 altered during the communication.

291

5) Now node $\mathrm{B}$ selects the big prime value $b$ and computes 292 the values of $T_{b}(x), K_{s}, H_{b}$, and $C_{b}$.

6) Node $\mathrm{B}$ sends the message $m b=\left\{H_{b}, C_{b}, T_{b}(x)\right\}$ to 294 node $\mathrm{A}$.

7) After getting the message $m b=\left\{H_{b}, C_{b}, T_{b}(x)\right\}$ from 296 node B, A computes the value of $K_{s}=T_{p b}(x)={ }_{297}$ $T_{p}\left(T_{b}(x)\right)$ by using $T_{b}(x)$ from message $m b$. Then, node 298 A decrypts $C_{b}$ with the key $K_{s}$, and compares the value 299 of PW from the decrypted message with its obtained 300 PW value from TTP. If there is a match, then node $\mathrm{A} 301$ concludes that B is an authenticated node.

8) Afterward, it checks the message integrity by computing 303 the hash value, and compares it with $H_{b}$. If there is 304 a match, then B concludes that the message was not 305 altered during the communication.

\section{6}

9) Finally, both the nodes A and B agree on an identical 307 session key $K_{S}$ and further communication is encrypted 308 and decrypted by session key $K_{S}$.

\section{B. Intervehicle Trust}

310

Intervehicle trust is composed of two main metrics: 1) direct ${ }_{311}$ trust and 2) indirect trust.

312

The interaction-based trust, i.e., (Direct $T(i, j)$ ), of the $j$ th 313 vehicle as evaluated by the $i$ th vehicle, is the ratio of honest 314 actions $\# H(i, j)$ to the total number of actions, i.e., both honest 315 and dishonest \#All $(i, j)$. It follows that the interaction-based 316 trust is calculated as:

$$
\operatorname{Direct} T(i, j)=\frac{\# H(i, j)}{\# \operatorname{All}(i, j)} \cdot\left[1-\frac{1}{H(i, j)+1}\right] .
$$

From (1), we can see that $1-(1 /[H(i, j)+1])$ increases 319 with respect to the increased number of honest actions in such 320 a way that several honest actions are needed to increase the ${ }_{321}$ interaction-based trust.

322

In our proposal, the intervehicle exchanged opinions (i.e., 323 Indirect trust) are sent together with the unencrypted part of ${ }_{324}$ exchanged data messages. To favor the opinions sourced by ${ }_{325}$ vehicles considered as trusted, the received recommendations 326 (opinions) sourced by a vehicle $k$ concerning the behavior of ${ }_{327}$ a vehicle $j$ [i.e., $\operatorname{Indirect} T_{k}(i, j)$ ] are combined with respect to 328 the honesty level of the recommender $k$, as follows: 329

$$
\operatorname{Indirect} T_{k}(i, j)=[\operatorname{Direct} T(i, k) \cdot \operatorname{Recom}(k, j)]^{\frac{1}{2}} \text {. (2) } 330
$$

Then, the different vehicles' recommendation about the jth ${ }_{331}$ vehicle are combined together to find the global vehicles' ${ }_{332}$ recommendation value for that vehicle $\mathrm{RV}(i, j)$, i.e.,

$$
\operatorname{Indirect} T(i, j)=\left[\prod_{|\operatorname{Recom}|}^{k} \operatorname{Indirect} T_{k}(i, j)\right]^{\frac{1}{|\operatorname{Recom}|}} .
$$

\section{Road Side Units Trust}

335

Simultaneously with the different intervehicle interactions, 336 whenever a vehicle joins the communication range of an RSU, 337 it sends its different neighbors overall trust to the road side 338 


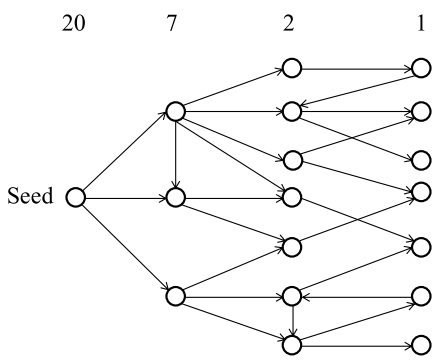

Fig. 5. Capacity assignment example.

339 unit. Afterward, the RSU combines all vehicles reports to build 340 a quasi-global evaluation of the behavior of vehicles moving 341 around.

342 Following (4), the roadside units compute their opinion ${ }_{343}$ regarding any vehicle $j$ through the combination of the reports 344 delivered by the other vehicles, i.e.,

$$
\mathrm{RR}(\mathrm{RSU}, j)=\left[\prod_{n}^{i} \operatorname{Tr}(i, j)\right]^{\frac{1}{n}}
$$

346 where $n$ represents the number of vehicles having previously ${ }_{347}$ evaluated the $j$ th vehicle.

\section{${ }_{348}$ D. Location-Related Trust}

349 TACASHI classifies the LRH of a given vehicle through 350 a similarity measurement between the current position and 351 the estimated position, based on their historical mobility pat352 terns [22]. Social events, such as soccer games, festivities, 353 and emergency cases are also taken into account for the path 354 estimation (see Fig. 4).

\section{E. Social Networks Trust: Using the Advogato Trust Metric} 356 to Identify Trustable People

${ }_{357}$ Various social networking aspects have been studied by an 358 online, free software developers community called Advogato. 359 This community, launched in 1999, has adopted a group-trust 360 metric trying to determine the largest set of honest peers, while 361 minimizing the influence of unreliable/dishonest ones [5]. 362 Advogato uses a social graph to represent the different peers 363 and relations in the network. Each peer in the graph represents 364 a user's account, whereas a directed edge represents a relation 365 (also called "certification").

366 The Advogato trust metric stands on the network flow. It 367 first assigns a "capacity" $C_{i}$ to every peer $i$, which represents a 368 nonincreasing function of the distance separating the peer $i$ and 369 the seed, as returned by the considered searching (breath-first 370 algorithm). For instance, "advogato.org" assigns a 20 capacity 371 for the seed, then 7 for the following two levels, 2 for peers 372 belonging to the third level, and so on (see Fig. 5).

373 Each node $\mathrm{A}$ is then divided into two sides, i.e., $\mathrm{A}-$ and $374 \mathrm{~A}+$, with a capacity -1 edge from A to the sink, and a capacity 375 of $\left(C_{i}-1\right)$ edge from $\mathrm{A}-$ to $\mathrm{A}+$, respectively. Finally, the 376 certification of A to B becomes an infinite-capacity edge from $377 \mathrm{~A}+$ to B- (see Fig. 6).

378 To find the maximum flow [24], Advogato is based on the ${ }_{379}$ Ford-Fulkerson algorithm (see Fig. 7). Since Ford-Fulkerson

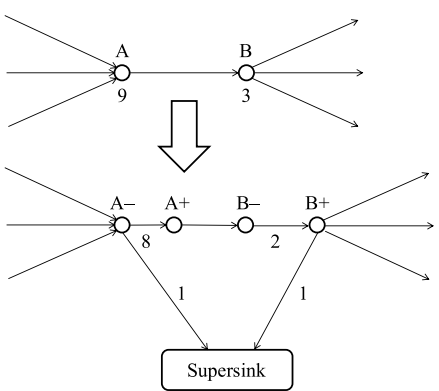

Fig. 6. Conversion into a single source, single sink.

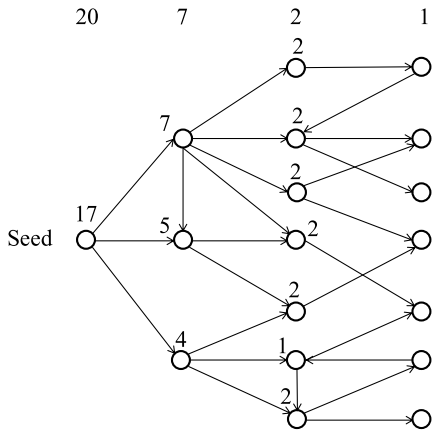

Fig. 7. Network flow computation.

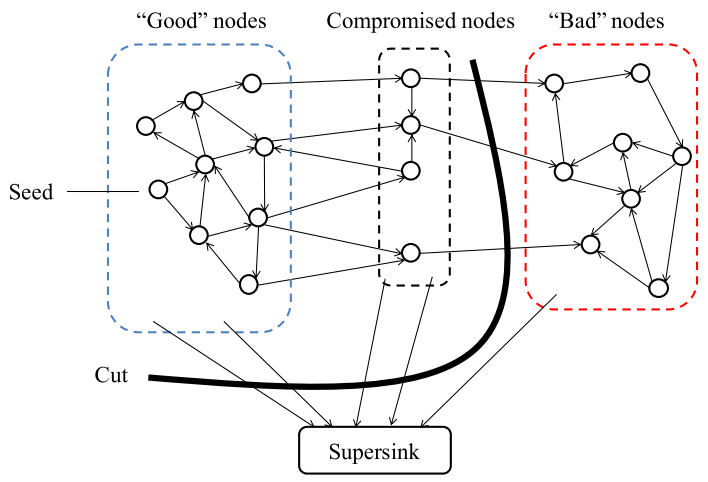

Fig. 8. Nodes classification.

selects the shortest increasing path from the current node to 380 the seed, any node having a flow from $x-$ to $x+$ possesses also 381 a flow from $x$ - to the sink. Ford-Fulkerson takes $O(|f+||E|), 382$ where $f$ is the maximum flow. In this graph, $f+$ is the number 383 of accepted peers.

Concerning the trusted accounts identification, an adversary 385 model should be defined first. Then, the minimum cut is cre- 386 ated to distinguish between trusted, doubted, and compromised 387 accounts, as shown in Fig. 8. The graph's minimum cut (i.e., 388 a partition of the nodes of a graph into two or more- $k$-cut- 389 disjoint subsets that are joined by at least one edge) is the one 390 that is minimal in some sense (trust value in our case). We note 391 that the Advogato trust metric has a wide range of applications, 392 meaning that edges and connections can be defined in different ${ }_{393}$ ways, including, for instance, communities, friendship, shared 394 posts, comments, or likes. 


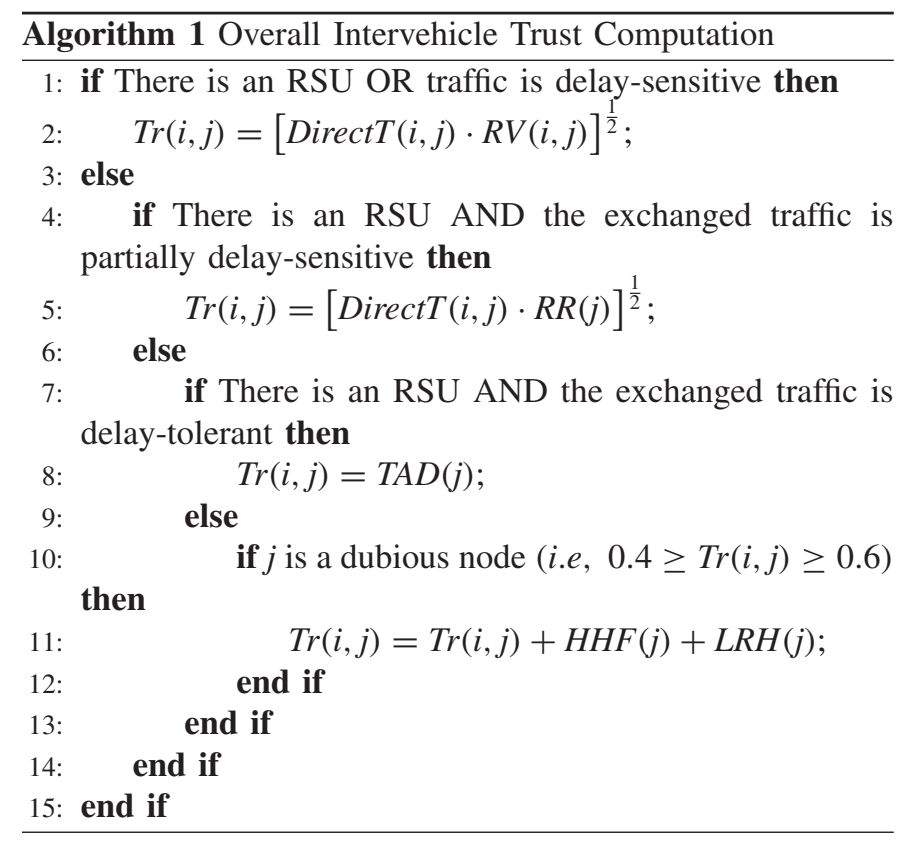

\section{TACASHI's Dishonesty Detection Process}

397

In addition to the direct and recommendation-based trust, 398 TACASHI involves also the driver's honesty factor based on 399 their OSN profiles. This information is received through the 400 trusted middleware, which for our case can be the TA, the 401 deployed RSUs, or even network operators. Furthermore, the 402 vehicles' LRH is also taken into account in the overall trust 403 evaluation.

404 If a vehicle has already demonstrated its honesty, and 405 thereby benefits from an high trust value, there is no need to 406 take the driver's honesty factor into account, and vice versa. 407 Thus, nodes requiring the human honesty factor as comple408 mentary data should be only those nodes whose behavior is 409 unclear/compromised.

${ }_{410}$ Depending on the OSNs, and having trust computed through 411 the Advogato trust metric, the TA matches, for each vehi412 cle identity, an honesty factor called honesty human factor ${ }_{413}$ (HHF), which refers to the human trust factor of the current 414 driver. This factor varies within the range of $[-0.5,-0.2]$ for 415 the drivers judged as bad, $[-0.2,0]$ for the drivers judged as 416 compromised, and $[0,+0,2]$ for the drivers judged as good. ${ }_{417}$ Whereas, the overall trust is in the range of $[0,1]$.

${ }_{418}$ In addition, using a path prediction algorithm [22], the LRH ${ }_{419}$ factor is also considered.

420 Similarly to the HHF, the LRH varies in the range of ${ }_{421}[-0.5,-0.2]$ for the positions judged as bad, $[-0.2,0]$ for the 422 positions that are compromised, and $[0,+0,2]$ for the posi${ }_{423}$ tions judged as good. Once the soliciting vehicles receive the ${ }_{424} \mathrm{HHF}$ and LRH for neighbors they have concerns about, the 425 trust computation will follow Algorithm 1. In this algorithm, ${ }_{426} \operatorname{Tr}(i, j)$ is the global intervehicle trust, $\operatorname{RV}(i, j)$ is the recom${ }_{427}$ mendation coming from a nearby vehicle, $\mathrm{RR}(\mathrm{RSU}, j)$ is the 428 recommendation requested and received from a nearby road 429 side unit, and, finally, $\operatorname{RT}(T A, j)$ is the TA evaluation about the $430 j$ th vehicle's honesty.
The trust evaluation $\operatorname{Tr}(i, j)$ is assessed after every update to ${ }_{431}$ keep it within the range $[0,1]$. Using this strategy, the number ${ }_{432}$ of dubious nodes will be reduced. Thus, a decision about the ${ }_{433}$ vehicles' trustiness can be made. The latter is made by using ${ }^{434}$ the different vehicles reports to generate a blacklist of the ${ }_{435}$ detected misbehaving vehicles, i.e.,

$$
\begin{aligned}
\mathrm{RSUBlacklist} & =\forall j \\
\frac{\operatorname{Card}(j / \operatorname{Tr}(i, j) \leq 0.5)}{\operatorname{Card}(\operatorname{RC}(j))} & \geq D \text { Threshold }
\end{aligned}
$$

where $D$ Threshold represents the threshold beyond which a ${ }_{439}$ vehicle is blacklisted. This threshold is compared with the ratio 440 of negative reports about the $j$ th vehicle to the total number ${ }_{441}$ of reports.

442

The TA's recommendations are in fact decisions that must 443 be followed by the different sublevels (RSUs and vehicles). ${ }_{444}$ It makes a decision $\operatorname{TAD}(j)$ about the $j$ th vehicle. TA deci- 445 sions are used only for nondelay-sensitive applications, as they ${ }_{446}$ involve all the lower level evaluations, thus implying additional ${ }_{447}$ computation delays. Therefore, the TA decision is computed ${ }_{448}$ according to

$$
\operatorname{TAD}(j)=\left[\prod_{n}^{i} \operatorname{RR}\left(\mathrm{RSU}_{i}, j\right)\right]^{\frac{1}{n}}
$$

where $n$ represents the number of RSUs having previously ${ }_{451}$ evaluated the $j$ th vehicle.

\section{Vi. Performance Evaluation}

Our proposal is implemented in the NS-2.35 simulator. In ${ }_{454}$ addition, we used the same dataset as in [25]. This dataset, 455 called Epinions [26], has 131828 nodes (users) and 841372456 edges (honest or malicious). We also consider that $30 \%$ of the ${ }_{457}$ edges represent a distrust relationship, and they are toward the 458 $10 \%$ and $20 \%$ vehicles considered as dishonest. Hence, we 459 considered in every case $10 \%$ of false evaluations (false pos- 460 itives). We selected the first 400 nodes that have more than ${ }_{461}$ 40 out-neighbors, and we randomly matched their identities 462 to 400 vehicle identities. Thus, every vehicle driver is repre- 463 sented by a node within the used dataset. Furthermore, in every 464 vehicle, we have four devices, being one of them assumed 465 unknown.

For VANET settings, the traffic is generated using the ${ }_{467}$ Citymob mobility model [27]. In our case, we used a $4 \mathrm{~km}^{2}{ }_{468}$ map of Laghouat city in Algeria. The generated vehicles path 469 of $80 \%$ of the vehicles to enable the paths prediction. For the 470 $20 \%$ remaining vehicles, half of them are moving toward pre- 471 defined positions called emergency location and event location 472 (i.e., hospital, soccer stadium, and so on), and the other half 473 are assumed to move to unpredictable positions. The scenario 474 has four randomly deployed RSUs. We run our simulation for 475 a duration of $1000 \mathrm{~s} 15$ times to reach the $95 \%$ confidence 476 level. In addition, the vehicles communication range is set 477 $300 \mathrm{~m}$ and they are moving with a speed varying in the range 478 of $[0,80] \mathrm{km} / \mathrm{h}$. Finally, ten randomly chosen vehicles send 479 four data packets of 256 bytes each every second. 480

In the following, we will compare the obtained dishon- 481 esty detection ratios to ones of RTM [18] and AD-IoV [28]. 482 


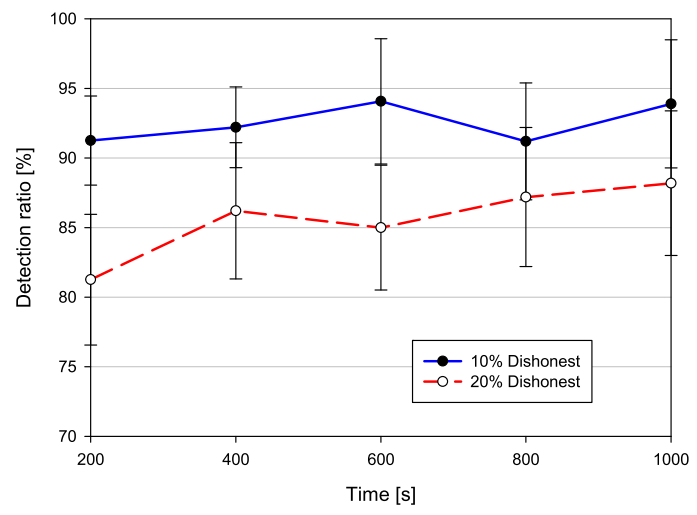

Fig. 9. Detection performance without the drivers honesty consideration.

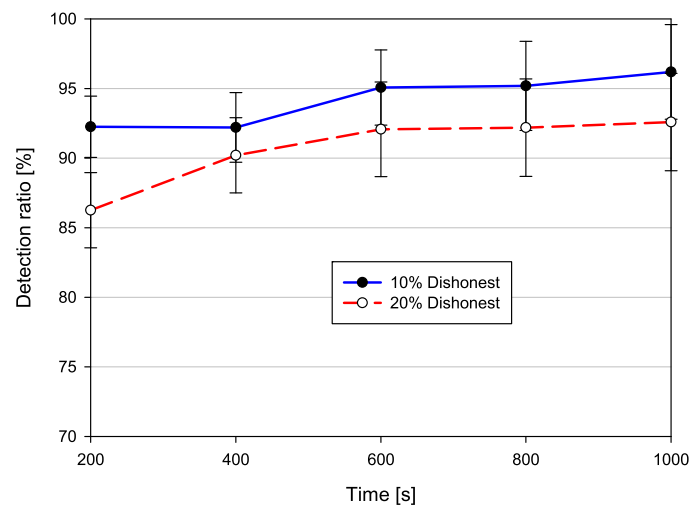

Fig. 10. Detection performance when considering the HF.

483 Afterward, we will analyze the generated error ratios with and 484 without the use of our proposed OSN-aided trust architecture.

For the detection performance we also studied both cases, 486 with and without human factor considerations. Fig. 9 rep487 resents the obtained detection ratio without using HHF for $48810 \%$ and $20 \%$ of dishonest vehicles with respect to time, 489 respectively. It shows that, although the average detection ratio 490 exceeds the $90 \%$ for $10 \%$ of malicious nodes, the confidence 491 interval is quite large, reaching the $5 \%$ at the end of the vari492 ous runs. This is mainly because of the doubtful behavior of 493 some peers that must be classified as behaving good or bad. 494 On the other hand, when the human factor is considered (see 495 Fig. 10), the detection ratio reaches up to $96 \%$ for $10 \%$ of 496 dishonest vehicles, and $93 \%$ for the $20 \%$ case, with clearly 497 more reduced confidence intervals.

498 Compared to the detection ratios achieved by RTM and 499 AD-IoV, both TACASHI versions with (i.e., TACASHI+) 500 and without (i.e., TACASHI-) driver's honesty consideration 501 achieved higher detection ratios. Even more, with TACASHI+ 502 the obtained detection ratios reach almost optimal perfor503 mance, as depicted in Fig. 11. This is mainly due to the 504 incorporation of OSN to enhance the trust establishment and, 505 thus, reduce the detection error ratios.

506 Confirming the previous results, the number of generated 507 false positives with respect of time is optimized by more than $5083 \%$, with more reduced confidence intervals compared to the 509 case where the driver factor is not considered (see Fig. 12). 510 However, the generated error ratio by both RTM and AD-IoV

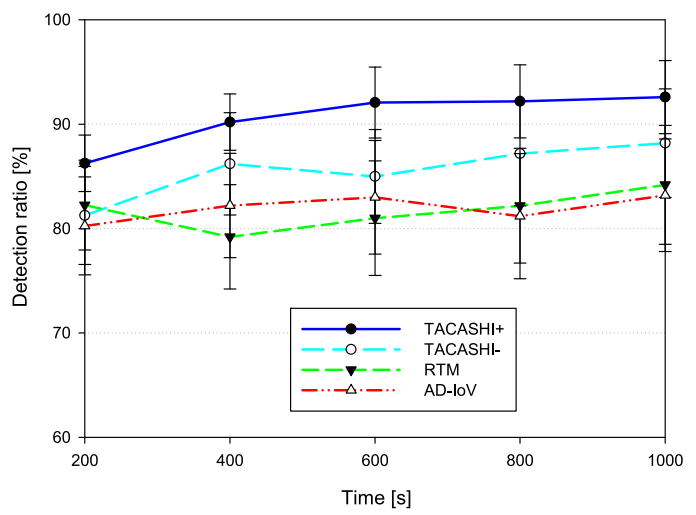

Fig. 11. Detection performance of TACASHI with and without considering the HF compared to RTM and AD-IoV.

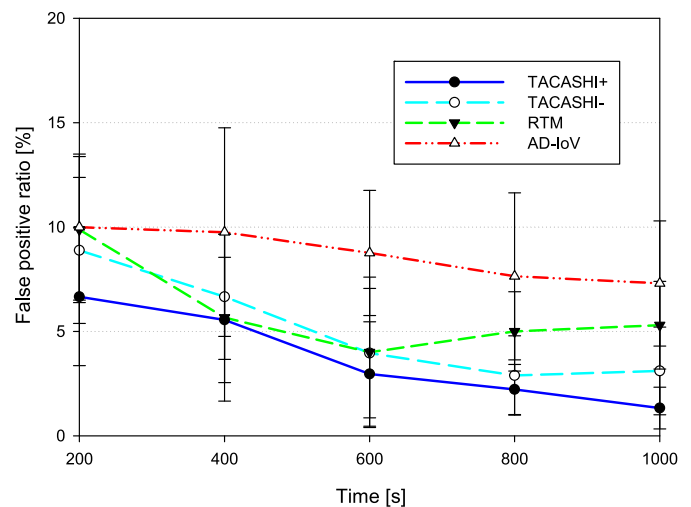

Fig. 12. Generated false positives by TACASHI with and without considering the HF compared to RTM and AD-IoV.

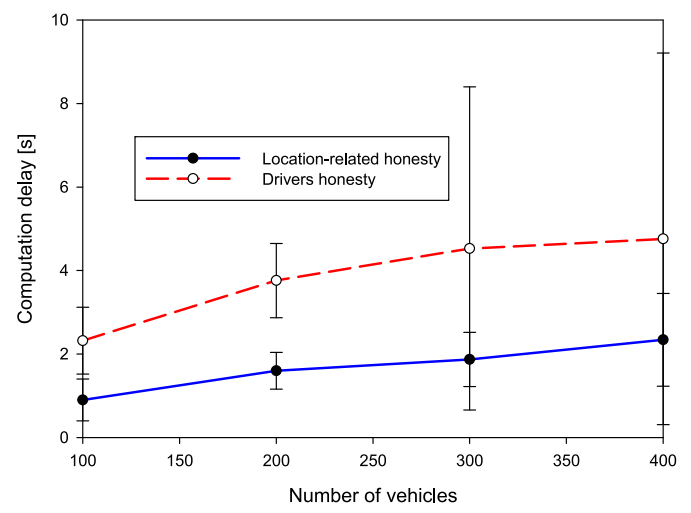

Fig. 13. TACASHI's introduced delay to compute HF and the LRH.

is quite high, reaching up to $10 \%$ for $\mathrm{AD}-\mathrm{IoV}$, which may ${ }_{511}$ cause some undesired situations. 512

Although the use of the OSNs and path prediction algo- 513 rithms through the trusted middleware has enhanced the 514 overall trust establishment, it is still prone to cause some 515 additional delay which becomes unacceptable for safety appli- 516 cations. Fig. 13 presents the required computation delay of the 517 drivers' honesty from OSNs and vehicles LRH through the 518 trusted middleware. It shows that, on average, and based on 519 the drivers honesty estimation, our proposal requires up to $5 \mathrm{~s} 520$ in the worst case. Indeed, this delay is not acceptable for IoV ${ }_{521}$ safety applications, but still it is considered reduced enough to 522 
523 prevent terrorist attacks or stolen vehicles. For the latter case, 524 simulation results show that we can decide whether the cur525 rent position of a given vehicle is normal or abnormal within 526 less than $2 \mathrm{~s}$ in the worst case.

\section{VII. CONCLUSION}

${ }_{528}$ IoV is composed of smart IP-based objects having connec529 tivity to both the Internet and to other vehicles, forming a 530 social network called SIoVs. Ensuring secure communication 531 among these vehicles and their embedded devices is an essen532 tial requirement of SIoV, especially when these communica533 tions are related to safety applications. In this paper, we aimed ${ }_{534}$ at the trust-driven security mechanism for SIoV and proposed 535 a novel trust-aware social in-vehicle and intervehicle commu536 nications architecture for SIoVs called TACASHI. In addition 537 to the intervehicle trust establishment and lightweight secure 538 in-vehicle communications, TACASHI also involves OSNs to 539 estimate the honesty of vehicles' drivers. Furthermore, the his540 torical mobility traces of the vehicles are stored and then 541 used to estimate their future path, while also considering 542 some exceptions, such as emergency situations and events. ${ }_{543}$ Simulation results demonstrate the performance of the pro544 posed TACASHI at ensuring high misbehavior detection ratios 545 clearly outperforms previous solutions known as RTM and 546 AD-IoV.

${ }_{547}$ As future work, we plan to add another social dimen548 sion to our architecture by also accounting for the trustiness 549 of unmanned aerial vehicles, and their interactions with the 550 vehicles and devices on the ground.
551

\section{REFERENCES}

[1] Y. Wang and F. Li, "Vehicular ad hoc networks," in Guide to Wireless Ad Hoc Networks. London, U.K.: Springer, 2009, pp. 503-525.

[2] M. Gerla, E.-K. Lee, G. Pau, and U. Lee, "Internet of Vehicles: From intelligent grid to autonomous cars and vehicular clouds," in Proc. IEEE World Forum Internet Things (WF-IoT), 2014, pp. 241-246.

[3] O. Kaiwartya et al., "Internet of Vehicles: Motivation, layered architecture, network model, challenges, and future aspects," IEEE Access, vol. 4, pp. 5356-5373, 2016.

[4] S. Mumtaz et al., "Cognitive vehicular communication for 5G," IEEE Commun. Mag., vol. 53, no. 7, pp. 109-117, Jul. 2015.

[5] R. Levien and A. Aiken, "Attack-resistant trust metrics for public key certification," in Proc. Usenix Security, 1998, p. 18.

[6] F. Xia, L. Liu, J. Li, J. Ma, and A. V. Vasilakos, "Socially aware networking: A survey," IEEE Syst. J., vol. 9, no. 3, pp. 904-921, Sep. 2015.

7] T. DuBois, J. Golbeck, and A. Srinivasan, "Predicting trust and distrust in social networks," in Proc. IEEE 3rd Int. Conf. Privacy Security Risk Trust (PASSAT) Soc. Comput. (SocialCom), 2011, pp. 418-424.

[8] Y. A. Kim and M. A. Ahmad, "Trust, distrust and lack of confidence of users in online social media-sharing communities," Knowl. Based Syst., vol. 37, pp. 438-450, Jan. 2013.

[9] S. Brin and L. Page, "Reprint of: The anatomy of a large-scale hypertextual Web search engine," Comput. Netw., vol. 56, no. 18, pp. 3825-3833, 2012.

10] J. Zhang, "A survey on trust management for VANETs," in Proc. IEEE It. Conf. Adv. Inf. Netw. Appl. (AINA), 2011, pp. 105-112.
[11] N. Yang, "A similarity based trust and reputation management frame- 578 work for VANETs," Int. J. Future Gener. Commun. Netw., vol. 6, no. 2, 579 pp. 25-34, 2013.

[12] N. Haddadou, A. Rachedi, and Y. Ghamri-Doudane, "Trust and exclu- 581 sion in vehicular ad hoc networks: An economic incentive model based 582 approach," in Proc. Comput. Commun. IT Appl. Conf. (ComComAp), 583 2013, pp. 13-18.

[13] S. Gurung, D. Lin, A. Squicciarini, and E. Bertino, "Information- 585 oriented trustworthiness evaluation in vehicular ad-hoc networks," in 586 Proc. Int. Conf. Netw. Syst. Security, 2013, pp. 94-108.

[14] C A. Kerrache, N. Lagraa, C. T Calafate, and A Lakas, "TFDD. A trust-based framework for reliable data delivery and dos defense in 589 VANETs," Veh. Commun., vol. 9, pp. 254-267, Jul. 2017.

[15] C. A. Kerrache, N. Lagraa, C. T. Calafate, J.-C. Cano, and P. Manzoni, 591 "T-VNets: A novel trust architecture for vehicular networks using the 592 standardized messaging services of ETSI ITS," Comput. Commun., 593 vol. 93, pp. 68-83, Nov. 2016.

[16] J. Contreras, S. Zeadally, and J. A. Guerrero-Ibanez, "Internet of 595 Vehicles: Architecture, protocols, and security," IEEE Internet Things 596 $J$., to be published.

[17] M. Hossain, R. Hasan, and S. Zawoad, "Trust-IoV: A trustworthy foren- 598 sic investigation framework for the Internet of Vehicles (IoV)," in Proc. 599 IEEE Int. Congr. Internet Things (ICIOT), 2017, pp. 25-32.

[18] F. Gai, J. Zhang, P. Zhu, and X. Jiang, "Trust on the Ratee: A trust 601 management system for social Internet of Vehicles," Wireless Commun. 602 Mobile Comput., vol. 2017, Dec. 2017, Art. no. 7089259.

[19] C. A. Kerrache, C. T. Calafate, J -C. Cano, N. Lagraa, and P. Manzoni, ${ }_{604}^{603}$ AQ6 "Trust management for vehicular networks: An adversary-oriented 605 overview," IEEE Access, vol. 4, pp. 9293-9307, 2016.

[20] A. M. Vegni and V. Loscri, "A survey on vehicular social networks," 607 IEEE Commun. Surveys Tuts., vol. 17, no. 4, pp. 2397-2419, 4th Quart., 608 2015.

[21] K. Zheng, Q. Zheng, P. Chatzimisios, W. Xiang, and Y. Zhou, 610 "Heterogeneous vehicular networking: A survey on architecture, chal- 611 lenges, and solutions," IEEE Commun. Surveys Tuts., vol. 17, no. 4, 612 pp. 2377-2396, 4th Quart., 2015.

[22] P. Lytrivis, G. Thomaidis, M. Tsogas, and A. Amditis, "An advanced 614 cooperative path prediction algorithm for safety applications in vehicular 615 networks," IEEE Trans. Intell. Transp. Syst., vol. 12, no. 3, pp. 669-679, 616 Sep. 2011.

[23] P. Bergamo, P. D'Arco, A. De Santis, and L. Kocarev, "Security 618 of public-key cryptosystems based on Chebyshev polynomials," IEEE 619 Trans. Circuits Syst. I, Reg. Papers, vol. 52, no. 7, pp. 1382-1393, 620 Jul. 2005.

[24] L. R. Ford and D. R. Fulkerson, "Maximal flow through a network," 622 Can. J. Math., vol. 8, no. 3, pp. 399-404, 1956.

[25] S. Al-Oufi, H.-N. Kim, and A. El Saddik, "A group trust metric for 624 identifying people of trust in online social networks," Expert Syst. Appl., 625 vol. 39, no. 18, pp. 13173-13181, 2012.

[26] J. Leskovec, D. Huttenlocher, and J. Kleinberg, "Signed networks in 627 social media," in Proc. SIGCHI Conf. Human Factors Comput. Syst., 628 2010, pp. 1361-1370.

[27] F. J. Martinez, J.-C. Cano, C. T. Calafate, and P. Manzoni, "CityMob: 630 A mobility model pattern generator for VANETs," in Proc. IEEE Int. 631 Conf. Commun. Workshops (ICC), 2008, pp. 370-374.

[28] S. Yang, Z. Liu, J. Li, S. Wang, and F. Yang, "Anomaly detection 633 for Internet of Vehicles: A trust management scheme with affinity 634 propagation," Mobile Inf. Syst., vol. 2016, Mar. 2016, Art. no. 5254141. 635 